\title{
Axion dark matter search experiment for the mass range of 6.62 to $7.04 \mu \mathrm{eV}$
}

\author{
Saebyeok Ahn* \\ Korea Advanced Institute of Science and Technology (KAIST) \\ E-mail: asb5229akaist.ac.kr
}

The CAPP-8TB axion dark matter search experiment for the mass range of $6.62 \mu \mathrm{eV}$ to $7.04 \mu \mathrm{eV}$ (the equivalent frequency range of $1.6 \mathrm{GHz}$ to $1.7 \mathrm{GHz}$ ) is being prepared as one of the CULTASK experiments at IBS/CAPP. The experiment utilizes the cavity haloscope technique introduced by Sikivie with a microwave resonant cavity of $3.5 \mathrm{~L}$ volume within a $8 \mathrm{~T}$ superconducting magnet at a cryogenic temperature of less than $0.1 \mathrm{~K}$. In this proceeding, details of the experiment configurations are discussed including the resonant cavity, cryogenics, magnet, tuning system and microwave receiver chain with high-electron mobility transistor (HEMT) amplifiers. The target of this effort is to be sensitive to the QCD axion band with two months of running

ICHEP 2018, International Conference on High Energy Physics

4-11 July 2018

Seoul, Republic of Korea

\footnotetext{
*Speaker.
} 
CAPP-8TB is an axion haloscope designed to be sensitive to cold dark matter axion in a mass range of $6.62 \mu \mathrm{eV}$ to $7.02 \mu \mathrm{eV}$. Inside a tunable copper cylindrical cavity of $3.5 \mathrm{~L}$ volume and $1.712 \mathrm{GHz}$ resonance frequency, the inverse Primakoff effect allows the presence of a resonant signal of axion coupled with virtual photons by a static magnetic field and the ambient axion field. The resonance frequencies of cavity are tuned by a cylindrical rod made of Alumina in order to have a bandwidth of search range, resulting in having $1.45-1.7 \mathrm{GHz}$ of resonance frequency of $\mathrm{TM}_{010}$ mode which has the highest geometrical factor among all cavity modes.
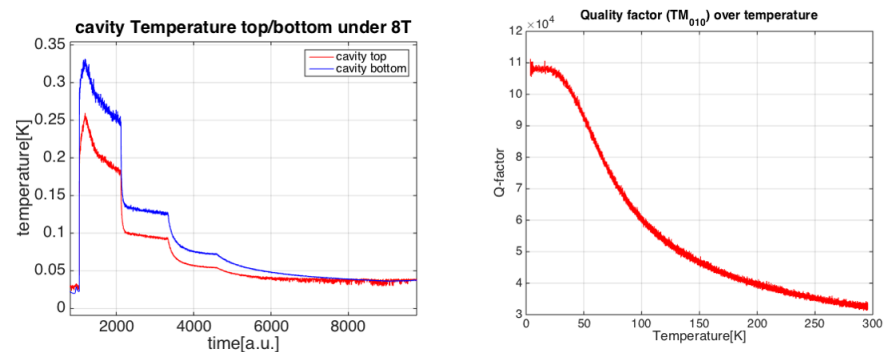

Figure 1: Cavity temperatures (left), and quality factor for the $\mathrm{TM}_{010}$ mode with temperature (right).

The cavity is attached to the stage of the lowest temperature in the dilution refrigerator. A result of preliminary measurement shows that the cavity temperature is saturated at around $40 \mathrm{mK}$, at which the $\mathrm{Q}$ factor of $\mathrm{TM}_{010}$ is around $10^{5}$. A superconducting magnet for the experiment stably provides an $8 \mathrm{~T}$ magnetic field at its center and the cavity is being placed where the volume average of fields inside the cavity is maximum, which is around 7.3 T. The signal power is pulled up to a measurable level by a cascaded RF chain having $130 \mathrm{~dB}$ of the total gain with two HEMT amplifiers. In the particular setup of the CAPP-8TB receiver chain, the noise temperature including those from the cavity and all passive devices is expected to be less than $2 \mathrm{~K}$. With all considered, the haloscope will be sensitive to four times the KSVZ QCD line. Two months of data taking will cover a search range of $1.6-1.7 \mathrm{GHz}$ with a target SNR of 5. After the first run, the first amplifier in the receiver chain will be replaced with a SQUID amplifier with nearly quantum-limited noise. As a result, the experimental sensitivity will be two times the KSVZ line with the same run period and search region.

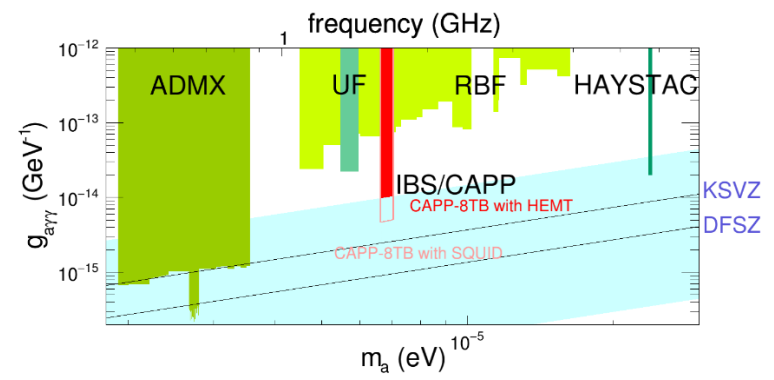

Figure 2: Expected sensitivities by the CAPP-8TB experiment.

\section{Acknowledgement}

This work was supported by IBS-R017-D1-2018-a00. 